\title{
ARTÍCULOS
}

\section{Aportes en la toma de decisiones para el manejo forestal con ganadería integrada del bosque de Prosopis caldenia del centro de Argentina}

\author{
Contributions in decision-making for forest management with integrated livestock in the Prosopis \\ caldenia forest of central Argentina
}

\author{
Marco Jesús Utello a*, Santiago Ignacio Fiandino a , Juan Carlos Tarico a , \\ Marcela Alejandra Demaestri a , José Omar Plevich a \\ *Autor de correspondencia: a Universidad Nacional de Río Cuarto, Facultad de Agronomía y Veterinaria, \\ Departamento de Producción Vegetal, Ruta Nacional 36, km 601, Río Cuarto (5800), Córdoba, Argentina, tel.: 54-358-4676509, \\ mutello@ayv.unrc.edu.ar
}

\begin{abstract}
SUMMARY
In Prosopis caldenia forests, currently, the main activity is cattle rearing. Almost all research focuses on the herbaceous component, losing sight of the value of the forest resource and the implications of its management on the forage resource. The objective of this work is to evaluate forage availability based on forest cover, apply a model of diameter classes that allows predicting the evolution of forest mass and establish the relationship between the evolution of forest cover and forage availability. For this, forage availability was measured under and outside the projection of woody canopies, in two covers: open (10-15 $\left.\mathrm{m}^{2} \mathrm{ha}^{-1}\right)$ and closed $\left(25-30 \mathrm{~m}^{2}\right.$ ha- $\left.{ }^{-1}\right)$. Afterwards, through a model of diameter classes, it was sought to predict how the parameters of forest mass would evolve. The results of the herbaceous component showed that, up to $15 \mathrm{~m}^{2} \mathrm{ha}^{-1}$ of basal area (BA), there is no significant decrease in forage availability (approximately 2,700 $\mathrm{kg} \mathrm{ha}^{-1}$ ). Regarding the forestry component, in a projected period of 10 years, $6.68 \mathrm{~m}^{3}$ ha $^{-1}$ would be obtained, representing $9.61 \%$ of total standing volume. The relationship between canopy coverage and BA showed increase of $3.18 \%$ per BA unit $\left(\mathrm{R}^{2}=0.96\right)$. This would allow projecting their participation by diameter class to propose improvement cuts that allow conducting these systems at coverage levels that do not significantly affect forage yield.
\end{abstract}

Key words: irregular structure, simulation, modeling, forage, pasture.

\section{RESUMEN}

En los bosques de Prosopis caldenia, actualmente, la principal actividad es la cría de ganado bovino. Casi la totalidad de las investigaciones se centran en el componente herbáceo perdiendo de vista el valor del recurso forestal y las implicancias de su manejo en el recurso forrajero. Este trabajo tiene como objetivos evaluar la disponibilidad forrajera en función de la cobertura boscosa, aplicar un modelo de clases diamétricas que permita predecir la evolución de la masa boscosa y establecer la relación entre la evolución del vuelo forestal y la disponibilidad forrajera. Para ello, se midió la disponibilidad forrajera bajo y fuera la proyección de copas de la leñosa, en dos coberturas: abierto (10-15 $\left.\mathrm{m}^{2} \mathrm{ha}^{-1}\right)$ y cerrado $\left(25-30 \mathrm{~m}^{2} \mathrm{ha}^{-1}\right)$. Luego mediante un modelo de clases diamétricas se buscó predecir cómo evolucionarían los parámetros de la masa forestal. Los resultados del componente herbáceo demostraron que, hasta 15 $\mathrm{m}^{2} \mathrm{ha}^{-1}$ de área basal (AB), no se producen disminuciones significativas en la disponibilidad forrajera (aproximadamente $2.700 \mathrm{~kg}^{2} \mathrm{a}^{-}$ $\left.{ }^{1}\right)$. En cuanto al componente forestal, en un lapso proyectado de 10 años, se obtendría $6,68 \mathrm{~m}^{3} \mathrm{ha}^{-1}$ y representa el $9,61 \%$ del volumen total en pie. La relación entre la cobertura de copas y el $\mathrm{AB}$, mostró un incremento de $3,18 \%$ por unidad de $\mathrm{AB}\left(\mathrm{R}^{2}=0,96\right)$. Esto permitiría proyectar su participación por clase diamétrica con el fin de proponer las cortas de mejoramientos que permitan conducir estos sistemas en niveles de cobertura que no afecten significativamente el rendimiento forrajero.

Palabras clave: estructura irregular, simulación, modelado, forraje, pastura.

\section{INTRODUCCIÓN}

La región Espinal abarca una superficie de $329.395 \mathrm{~km}^{2}$ bordeando la pampa argentina. Las tierras forestales cubren el 7,5\% del territorio y se concentran en el distrito del caldén (Prosopis caldenia Burk.) (SAyDS, 2007). El aprovechamiento forestal se desarrolló fuertemente durante la primera mitad del siglo XX. Sin embargo, en la actualidad la actividad forestal es marginal y la principal actividad económica desarrollada en los bosques es la cría de ganado va- 
cuno, que utiliza en forma casi exclusiva el forraje que crece en el sotobosque (SAyDS 2007). Por lo general, el manejo ganadero en la región es extensivo, con cargas animales inadecuadas, poca división de potreros y sin descansos estacionales (Dussart et al. 1998). Este tipo de manejo favorece la invasión de leñosas, que se ve potenciada por el efecto del bovino como agente dispersante (Pelaez et al. 1992).

Casi la totalidad de las investigaciones en bosque de $P$. caldenia se centran en el componente herbáceo (Gabutti et al. 1999, Esterlich et al. 2005, Morici et al. 2009) perdiendo de vista el valor del recurso forestal y las implicancias del manejo de este en el recurso forrajero. Tal es el caso del estudio realizado por Roman et al. (2015) en el noroeste de la ecorregión del espinal, quienes encontraron que la tasa de crecimiento de las especies herbáceas bajo la copa del estrato leñoso, fue superior a aquella medida en posiciones de cielo abierto. Sin embargo, la producción de biomasa herbácea no fue asociada a variables de densidad del estrato arbóreo y, en consecuencia, no se puede predecir qué efectos tendrían diferentes técnicas de manejo forestal sobre la misma, lo cual limita fuertemente la toma de decisiones.

Conocer la dinámica del crecimiento del vuelo forestal y su efecto sobre el estrato herbáceo resulta indispensable para mejorar la toma de decisiones a la hora de conducir este tipo de sistemas mixtos. Si bien existen estudios que involucran la dinámica de crecimiento y las tasas de regeneración del bosque de $P$. caldenia (Dussart et al. 1998, Bogino y Villalba 2008), los mismos no tuvieron como fin generar modelos de ordenación que proyecten la evolución del vuelo forestal para su manejo y aprovechamiento. La falta de modelos de ordenación es una limitante para la promoción del aprovechamiento y conservación del bosque, ya que los usuarios de los recursos requieren bases técnicas antes de implementar actividades económicas de aprovechamiento (Coirini 2013).

En masas irregulares en equilibrio el mismo número de pies que abandonan una clase diamétrica debido a la mortalidad, crecimiento o entresaca, son sustituidos por un número equivalente procedente de la clase diamétrica inferior en el período correspondiente entre dos intervenciones. La curva de equilibrio o distribución diamétrica ideal ha sido uno de los modelos biométricos más estudiados en el campo de las ciencias forestales (Madrigal 1994). Es por ello, que el diámetro del fuste es la variable más usada en las decisiones de manejo de bosques irregulares (Iturre et al. 2017). A su vez, los modelos de distribuciones diamétricas pueden resultar muy útiles en la proyección del cambio de frecuencia por categorías. Constituye un método relativamente simple para estimar el crecimiento y producción utilizando el incremento en diámetro de los árboles y otras variables referidas a la masa. Existe un gran número de antecedentes en modelos y propuestas de aprovechamiento del bosque nativo para diferentes regiones (Beltrán et al. 2018, Urbano et al. 2018). Para citar algunos ejemplos, está el sistema STAND aplicado por Vargas et al. (2008) en rodales irregulares y mixtos de la región de El Salto, Durango, México. También el Forest Vegetation Simulator (FVS), ampliamente aplicado en Estados Unidos. Se cuenta en bosques del chaco semiárido con la experiencia de la aplicación del simulador MOSIMAFO (Iturre et al. 2017).

En la ecorregión del Espinal, al presente no se citan experiencias en simulaciones del crecimiento del vuelo forestal y, menos aún, el empleo de modelos de clases diamétricas, como en el caso de la región chaqueña (Sanquetta et al. 1999, Araujo e Iturre 2014, Iturre et al. 2017). La principal causa radica en la dificultad de obtener los incrementos diamétricos en pies de diferentes edades, debido a la falta de inventarios forestales continuos en parcelas permanentes.

El siguiente trabajo tiene como objetivo general el de abordar en forma integral el estudio del componente forestal y herbáceo para aportar elementos en la toma de decisiones en el manejo del bosque de P. caldenia. Como objetivos específicos se considera: (a) evaluar la disponibilidad forrajera en términos de cantidad y calidad en función de la cobertura boscosa, (b) la aplicación de un modelo de clases diamétricas que permita predecir cómo evolucionarían los parámetros de la masa en un bosque de $P$. caldenia en relación a una distribución diamétrica ideal y (c) establecer la relación entre la evolución del vuelo forestal y la disponibilidad forrajera. Para tal fin, se realizaron inventarios de los recursos forestales y forrajeros.

\section{MÉTODOS}

Descripción y ubicación de la zona de estudio. El caldenal es un bosque xerófilo dominado por $P$. caldenia y es la formación arbórea predominante del distrito del caldenal dentro de la región fitogeográfica del Espinal. Su estructura, generalmente se presenta asociado con otras leñosas arbóreas como algarrobo (Prosopis flexuosa DC), chañar (Geoffroea decorticans Burkart) y sombra de toro (Jodina rhombifolia Hook. et Arn. Reissek) que es acompañado por un estrato arbustivo donde se destacan Schinus fasciculata (Griseb.), Celtis ehrenbergiana (Klotzsch) Liebm. y Condalia microphylla Cav. Presenta un estrato herbáceo denso compuestos por gramíneas perennes mixtas (Anderson et al. 1970).

La región de estudio se localiza 30 kilómetros al oeste de la localidad de Villa Huidobro, en el sudoeste de la provincia de Córdoba, Argentina. Se caracteriza climáticamente como semiárida en transición a subhúmeda de régimen monzónico. La temperatura media anual es de $16,6^{\circ} \mathrm{C}$. Enero es el mes más cálido con una media de $24{ }^{\circ} \mathrm{C}$, y julio el más frío con una temperatura media de $9{ }^{\circ} \mathrm{C}$. La precipitación media anual es de $893 \mathrm{~mm}$, aunque presenta gran variabilidad interanual (valores climatológicos medios 1981-2010 de las estadísticas del Servicio Meteorológico Nacional).

Definición del módulo silvopastoril. El estudio se realizó en el establecimiento "Estancia Ralicó" (34 54'25" S; $64^{\circ} 50^{\prime} 03$ " O) que posee una superficie de 15.300 ha, de la cuales 9.000 ha corresponden a bosque de $P$. caldenia en 
distinto grado de cobertura y conservación. Históricamente el establecimiento estuvo dividido en potreros de aproximadamente 600 ha. A partir del año 2016, se implementó un sistema de pastoreo rotativo de alta carga instantánea. Para ello, se dividió un lote de 600 ha en 30 parcelas de 20 ha. Las divisiones se realizaron mediante alambre eléctrico buscando espacios dentro del monte sin abrir picadas. El pastoreo se lleva a cabo introduciendo altas cargas, de entre 900 y 1.200 animales por parcela, en lapsos de 3 a 4 días, una vez al año. La cantidad de animales y la duración del tiempo se ajusta en función de la disponibilidad forrajera. Esta alta carga instantánea obliga a que animal no seleccione y consuma por completo la biomasa herbácea, lo que se verificó por la observación de un escaso o nulo remanente de pastoreo. Al comienzo de los ensayos fue necesario suplementar con rollos de heno de alfalfa para estimular a que los animales aprovechen el forraje de escasa calidad como resultados del subpastoreo del manejo anterior.

Inventario forestal. Para la caracterización del bosque se realizó un inventario forestal siguiendo los lineamientos del Primer Inventario Forestal Nacional (PIFN) (SAyDS 2007). Se establecieron 43 parcelas circulares de $500 \mathrm{~m}^{2}$, con un radio de 12,62 m, donde se midieron los diámetros a altura de pecho (dap), el reclutamiento, la altura total y la cobertura de copa.

Dentro de cada parcela se contemplaron todos los árboles que al menos poseían uno de sus fustes con un dap igual o mayor a $10 \mathrm{~cm}$; todos aquellos individuos con dap inferiores a ese valor fueron considerados parte del reclu- tamiento o regeneración del bosque. Los individuos con dap $\geq 10 \mathrm{~cm}$ fueron ordenados en clases diamétricas cuyo intervalo de clase fue de $5 \mathrm{~cm}$ y, a partir de ellos, se calculó el área basal $(\mathrm{AB}) \mathrm{en}^{2} \mathrm{ha}^{-1} \mathrm{y}$ se realizó una zonificación del bosque en dos estructuras: 1 . Bosque cerrado y 2 . Bosque abierto. A faltas de investigaciones locales que definan con precisión la densidad de un bosque abierto o cerrado, se estableció el límite de $15 \mathrm{~m}^{2} \mathrm{ha}^{-1}$ de AB. Por debajo se consideró bosque abierto y, por encima bosque cerrado. La definición de dicho valor se hizo a partir de lo propuesto por SAyDS (2007) donde establece valores de área basal promedios para bosque abierto y cerrado en el distrito del caldenal.

La regeneración se clasificó según tres clases de la siguiente manera: clase 1: renovales menores de $1,30 \mathrm{~m}$ de altura; clase 2: renovales mayores de $1,30 \mathrm{~m}$ de altura y menores de $5 \mathrm{~cm}$ de dap y; clase 3: renovales mayores de $1,30 \mathrm{~m}$ de altura y con dap entre 5 y $10 \mathrm{~cm}$.

Para la obtención de la altura total (h), que corresponde a la distancia desde el suelo hasta el extremo superior del árbol, se utilizó hipsómetro de Suunto. Para la medición de los diámetros de copas (DC) se proyectaron sus límites utilizando una regla telescópica de $4 \mathrm{~m}$. Luego con cinta métrica se midieron los radios hacia los cuatro puntos cardinales. En la medición de estas dos variables (h y DC) se incluyeron individuos de todas las clases diamétricas en cada parcela.

La composición específica a nivel de rodal fue evaluada definiendo los valores relativos en número de pies (densidad) y área basal (AB). Se pudo determinar una marcada dominancia de $P$. caldenia (cuadro 1) sobre el resto de las

Cuadro 1. Variables descriptivas medias y desvíos estándar en dos coberturas boscosas: abierto $\left(\mathrm{AB} \leq 15 \mathrm{~m}^{2}\right.$ ha $\left.{ }^{-1}\right)$ y cerrado $\left(\mathrm{AB} \geq 15 \mathrm{~m}^{2}\right.$ $\mathrm{ha}^{-1}$ ), de la estancia Ralicó (Provincia de Córdoba, Argentina). D: densidad (árboles ha $\left.{ }^{-1}\right), \mathrm{AB}$ : área basal ( $\mathrm{m}^{2}$ ha $\left.{ }^{-1}\right), \%$ D: valor relativo del número de pies ocupado por cada especie, y \% AB: valor relativo de área basal ocupado por cada especie. $\overline{\mathrm{X}}$ : media y $\mathrm{S}$ : desvío estándar.

Mean descriptive variables and standard deviations in two forest coverings: open $\left(A B \leq 15 \mathrm{~m}^{2} \mathrm{ha}^{-1}\right)$ and closed $\left(\mathrm{AB} \geq 15 \mathrm{~m}^{2}\right.$ ha-1), from Ralicó Farm (Córdoba Province, Argentina). Where: D: density (trees ha $\left.{ }^{-1}\right)$, AB: basal area $\left(\mathrm{m}^{2} \mathrm{ha}^{-1}\right), \%$ D: relative value of the number of feet occupied by each species, and \% AB: relative value of basal area occupied by each species. $\overline{\mathrm{X}}$ : mean and S: standard deviation.

\begin{tabular}{|c|c|c|c|c|c|c|}
\hline \multirow{2}{*}{ Tipo de bosque } & \multirow{2}{*}{ Variable } & \multicolumn{5}{|c|}{ Especie } \\
\hline & & P. caldenia & P. flexuosa & G. descorticans & S. fasciculata & J. rhombifolia \\
\hline & & $\overline{\mathrm{x}} \pm \mathrm{S}$ & $\overline{\mathrm{X}} \pm \mathrm{S}$ & $\overline{\mathrm{X}} \pm \mathrm{S}$ & $\overline{\mathrm{x}} \pm \mathrm{S}$ & $\overline{\mathrm{x}} \pm \mathrm{S}$ \\
\hline \multirow{5}{*}{ Abierto } & $\mathrm{D}$ & $165 \pm 147,67$ & $16 \pm 41,85$ & $6 \pm 14,65$ & $9 \pm 16,51$ & $3 \pm 7,33$ \\
\hline & $\mathrm{AB}$ & $7,68 \pm 4,05$ & $0,41 \pm 0,82$ & $0,17 \pm 0,57$ & $0,23 \pm 0,45$ & $0,06 \pm 0,21$ \\
\hline & $\% \mathrm{D}$ & $83,57 \pm 16,76$ & $5,83 \pm 12,59$ & $3,58 \pm 8,05$ & $5,86 \pm 10,15$ & $1,16 \pm 2,84$ \\
\hline & $\% \mathrm{AB}$ & $89,95 \pm 11,46$ & $4,56 \pm 9,63$ & $1,69 \pm 4,88$ & $3,2 \pm 6,78$ & $0,6 \pm 2,03$ \\
\hline & $\mathrm{D}$ & $312,38 \pm 147,44$ & $15,24 \pm 38,94$ & $20,95 \pm 41,22$ & $27,62 \pm 64,34$ & $0,95 \pm 4,36$ \\
\hline \multirow{3}{*}{ Cerrado } & $\mathrm{AB}$ & $21,47 \pm 8,52$ & $0,54 \pm 1,33$ & $0,36 \pm 0,71$ & $0,67 \pm 1,36$ & $0,01 \pm 0,05$ \\
\hline & $\% \mathrm{D}$ & $84,75 \pm 21,07$ & $3,11 \pm 6,81$ & $5,14 \pm 10,61$ & $7,01 \pm 14,44$ & $0,3 \pm 1,36$ \\
\hline & $\% \mathrm{AB}$ & $91,37 \pm 14,4$ & $3,25 \pm 8,09$ & $1,73 \pm 4,1$ & $3,63 \pm 6,99$ & $0,03 \pm 0,13$ \\
\hline
\end{tabular}


especies donde, el porcentaje relativo de ocupación promedio fue de $90 \%$ del área basal. El resto de la composición relativa se repartió entre P. flexuosa, G. descorticans, $S$. fasciculata y en menor proporción por J. rhombifolia, la cual suele aparecer como individuos dispersos. Por consiguiente, la modelación y el análisis de la cobertura se abordó desde la especie principal (P. caldenia) por ser la que mayor impacto tendría en el recurso forrajero y en el aprovechamiento forestal.

El estrato arbustivo estuvo integrado mayoritariamente por S. fasciculata, C. ehrenbergiana y C. microphylla. La aparición de estas especies no es continua. Existen zonas de considerable abundancia y otras relativamente escasas.

Medición de la biomasa herbácea. Establecida la estratificación del bosque en abiertos $\left(\mathrm{AB} \leq 15 \mathrm{~m}^{2} \mathrm{ha}^{-1}\right)$ y cerrados $\left(\mathrm{AB} \geq 15 \mathrm{~m}^{2} \mathrm{ha}^{-1}\right)$, la disponibilidad del estrato herbáceo se determinó por medio de muestreos completamente aleatorizados en las siguientes condiciones: 1 . bajo copa en bosque cerrado, 2. entre copas en bosque cerrado, 3 . bajo copa en bosque abierto y 4 . entre copas en bosque abierto. Se tomaron 16 repeticiones por condición. Los muestreos se realizaron previo a la entrada de los animales y el material recolectado comprende al acumulado entre dos pastoreos, de acuerdo al sistema de pastoreo expuesto anteriormente, corresponde a un año de crecimiento.

Las situaciones de bosque cerrado alcanzaron en promedio $27,5 \mathrm{~m}^{2} \mathrm{ha}^{-1}$ de $\mathrm{AB}$ y se encontraron pocos intersticios de espacio sin proyección de copas. Los bosques abiertos presentaron en promedio $12,5 \mathrm{~m}^{2} \mathrm{ha}^{-1}$ de AB. El tamaño de muestreo para el estrato herbáceo fue de 0,25 $\mathrm{m}^{2}$. En laboratorio se identificaron las especies que integraban las muestras y se determinó los porcentajes en término de ocupación. Luego se llevaron a estufa $\left(60^{\circ} \mathrm{C}\right)$ de aire forzado hasta peso constante. Posteriormente se realizó un análisis de calidad por condición, donde se terminó: proteína cruda, digestibilidad de la fibra en detergente neutro (FDN), total de nutrientes digestibles (TND), carbohidratos no fibrosos (CNF) y valor relativo del forraje (VRF). El análisis de calidad no tuvo como fin encontrar diferencias estadísticas entre condiciones, ya que es conocida la gran variación temporal del pastizal, además de la selección por parte de los animales. Lo que se persiguió fue determinar dentro de qué valores se encontraba en relación al aprovechamiento nutricional de la biomasa.

Obtención de un modelo de distribución diamétrica. Para establecer el modelo de distribución diamétrica del componente leñoso bajo estudio, se siguieron los lineamientos propuestos en el modelo, o Ley de De Liocourt (Madrigal 1994):

$$
N_{i}=N_{\max }(1+a)^{\left(D_{\max }-D_{i}\right) / d}
$$

Donde, Ni: $\mathrm{N}^{0}$ de pies de la clase i; Di: Diámetro medio de la clase i; Nmax: $\mathrm{N}^{\circ}$ de pies de la clase de diámetro máximo; Dmax: Diámetro medio de la clase de diámetro máximo; d: Intervalo de clases diamétricas; y a: constante de modelo.

El modelo exponencial puede formularse también con la expresión [2]:

$$
\mathrm{Ni}=\mathrm{k} \cdot \mathrm{e}^{-\mathrm{q} * \mathrm{Di}}
$$

Donde k y q son constantes, siendo $\mathrm{q}=1 / \mathrm{d} * \ln (1+\mathrm{a})$.

La función exponencial [2] se transformó en una función lineal aplicando logaritmos [3]:

$$
\ln \mathrm{y}=\ln \mathrm{k}-\mathrm{q} * \ln \mathrm{e} \mathrm{Di}
$$

Para luego expresarse como:

$$
\mathrm{Z}=\beta_{0}+\beta_{1} * \mathrm{X}_{\mathrm{i}}
$$

Donde: $Z$ = número de pies para un valor área basimétrica $(\mathrm{AB})$, el diámetro máximo (Dmax) y el cociente de De Liocourt "q". El termino Xi reemplaza a Di.

Esta función transformada permitió establecer relaciones que posibilitaron calcular el coeficiente $\beta_{1}$ para un valor de "q" elegido y de $\beta_{0}$ para una determinada área basal (Araujo 2014).

El cociente entre el número de individuos de clases diamétricas consecutivas es igual a la constante "q", por lo tanto, se puede expresar como:

$$
\frac{\mathrm{Ni}}{\mathrm{Ni}+1}=\frac{\mathrm{e}^{(\beta 0+\beta 1 * \mathrm{Xi})}}{\mathrm{e}^{(\beta 0+\beta 1 * \mathrm{Xi}+1)}}=q
$$

Multiplicando los términos de la ecuación, se obtiene:

$$
\mathrm{q} * \mathrm{e}^{(\beta 0+\beta 1 * \mathrm{Xi+1})}=\mathrm{e}^{(\beta 0+\beta 1 * \mathrm{Xi})}
$$

Aplicando logaritmos y simplificando:

$$
\begin{gathered}
\ln \mathrm{q}+(\beta 0+\beta 1 * X i+1) \ln \mathrm{e}=(\beta 0+\beta 1 * X i) \ln \mathrm{e} \\
\ln \mathrm{q}=\beta 0+\beta 1 * X i-\beta 0-\beta 1 * X i+1 \\
\ln \mathrm{q}=\beta 1 * X i-\beta 1 * X i+1 \\
\ln \mathrm{q}=\beta 1(X i-X i+1)
\end{gathered}
$$

Despejando $\beta 1$ :

$$
\beta 1=\frac{\operatorname{Lnq}}{\mathrm{Xi}-\mathrm{Xi}+1}
$$

Con esta expresión se calculó el nuevo valor de $\beta 1$ para un determinado valor de "q". De la misma manera, el área basal (AB), expresada en $\mathrm{m}^{2} \mathrm{ha}^{-1}$, se define por:

$$
\mathrm{AB}=\frac{\pi * D_{1}^{2}}{40.000} * f_{1}+\frac{\pi * D_{2}^{2}}{40.000} * f_{2}+\cdots \frac{\pi * D n^{2}}{40.000} * f n
$$


En que $D l^{2}, . ., D n^{2}$ representan el menor y mayor diámetro de la distribución y $f 1, . ., f n$ son las frecuencias respectivas.

Despejando el término ( $p$ / 40.000), el factor común $e^{\beta 0}$ y reemplazando las frecuencias $\mathrm{f}$ por su valor $\left[\mathrm{e}^{\left(\mathrm{b} 0+\mathrm{bl} \mathrm{Xi}_{\mathrm{i}}\right.}\right] \mathrm{se}$ obtuvo:

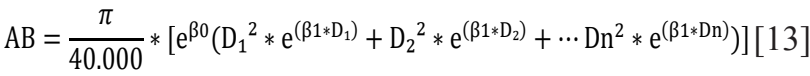

Donde:

$\mathrm{e}^{\beta 0}=\frac{\mathrm{AB} * 40.000}{\pi *\left(\mathrm{D}_{1}{ }^{2} * \mathrm{e}^{\left(\beta 1 * \mathrm{D}^{1}\right)}+\mathrm{D}_{2}{ }^{2} * \mathrm{e}^{\left(\beta 1 * \mathrm{D}^{2}\right)}+\cdots+\mathrm{Dn}^{2} * \mathrm{e}^{(\beta 1 * \mathrm{Dn})}\right)}[14]$

Finalmente, aplicando logaritmo neperiano se llegó a la expresión que relaciona el coeficiente $\beta 0$ con el área basal AB:

$$
\beta 0=\operatorname{Ln} *\left[\frac{\mathrm{AB} * 40.000}{\pi *\left(\Sigma \mathrm{Di}^{2} * \mathrm{e}^{(\beta 1 * \mathrm{Di})}\right)}\right]
$$

La base del método es la determinación de partes proporcionales de área basal para las diferentes clases, que luego se usan para calcular un área basal correcta, distribuyendo el nivel deseado de área basal en las respectivas clases. Luego, el área basal elegida es transformada en número de árboles para construir la distribución diamétrica.

Por consiguiente, para definir la distribución equilibrada se seleccionaron los valores de q, $\mathrm{AB}$ y diámetro máximo (Dmax). El valor de $\mathrm{q}=1,46$, empleado para la construcción de la curva de equilibrio, se obtuvo de ajustes previos en bosque de buen estado de cobertura. $\mathrm{El} \mathrm{AB}$, se fijó en $15 \mathrm{~m}^{2} \mathrm{ha}^{-1}$, esto guarda relación con los resultados del apartado de la biomasa forrajera.

Se utilizó un Dmax de $50 \mathrm{~cm}$, establecido a partir de que, bajo el régimen de aprovechamiento de entresaca, se corta la última clase llegando a un diámetro mínimo de corta de $45 \mathrm{~cm}$. En la gran mayoría de los Prosopis spp., cuando el individuo alcanza ese dap garantiza un buen desarrollo, capaz de producir abundantes semillas y un buen rendimiento en aserradero. Además, como lo plantea Bogino y Villalba (2008), entorno a ese diámetro el incremento corriente se cruza con el incremento medio estableciendo el momento óptimo de aprovechamiento desde el punto de vista forestal.
Proyección del crecimiento forestal. La modelación del crecimiento fue realizada a través de un modelo de clases diamétricas, utilizando como base la distribución diamétrica inicial obtenida del inventario. El modelo permitió proyectar la evolución del número de pies por clase diamétrica. Dado el corto período de tiempo de la modelación (10 años) se asumió que todos los árboles sobrevivirían al período considerado.

Asumiendo la propuesta de Silva (1989), la fracción de árboles que se mueve anualmente a lo largo de todo el intervalo de clases, debido al crecimiento diametral, fue estimada por un índice de crecimiento (IC), calculado por la siguiente fórmula:

$$
\mathrm{IC}=\mathrm{Ic} * \mathrm{P} / \mathrm{a}
$$

Donde, $I C$ : Índice de crecimiento. $I c$ : Incrementos corriente en diámetro de la clase. $P$ : Número de años del período considerado. $a$ : Amplitud de la clase de diámetro.

Para el cálculo del $I c$ se emplearon los datos obtenidos en el trabajo de Bogino y Villalba (2008), donde se establece el crecimiento radial y tiempos de rotación de $P$. caldenia en el centro de la Argentina. Con los datos dendrocronológicos del sitio "El Liebral", próximo al estudiado, a partir del ajuste de una función de tres tramos, ecuación [17], se obtuvieron los incrementos corrientes (Ic) por clase diamétricas:

Conociendo las existencias medias anuales en número de individuos por clase diamétrica y el IC, fue posible calcular el número de años necesarios para que todos los pies de una clase pasen a la siguiente y proyectar la distribución diamétrica.

Otro punto importante a considerar en el modelado fue el ingreso o reclutamiento que se introducirían a la primera clase diamétrica. A falta de experiencias locales, las cantidades mínimas y las cantidades ideales de árboles que deben permanecer en la regeneración por hectárea se determinaron a base de experiencias hechas en bosques de estructura similar del Chaco semiárido (Coirini 2013). En el caso de bosques de $P$. caldenia, la cantidad mínima debería estar entorno a los 150 a 200 árboles de futura cosecha por hectárea (Coirini 2013). La cantidad de ejemplares inventariados en la regeneración fue de 234 individuos ha- ${ }^{-1}$. A esos 234 pies se los multiplicó por el IC de la clase y arrojó un valor de 23,4 pies ha ${ }^{-1}$ año ${ }^{-1}$. En relación con esto, se formuló la hipótesis de que ingresarán 23,4 individuos por

$$
\text { Ic }=2,92+\left\{\begin{array}{lr}
(0,87 * \text { dap }) & \text { dap } \leq 4,44 \mathrm{~cm} \\
((0,87 * 4,44)+(\text { dap }-4,44) *-0,07) & 4,44 \mathrm{~cm}<\text { dap } \leq 32,89 \mathrm{~cm} \\
((0,87 * 4,44)+(32,89-4,44) *-0,07-0,05 *(\text { dap }-32,89)) & \text { dap }>32,89 \mathrm{~cm}
\end{array}\right.
$$

Donde, Ic: incremento corriente anual $(\mathrm{mm})$. dap: diámetro a altura de pecho $(\mathrm{cm})$. 
año (tasa de reclutamiento). En el Chaco semiárido Iturre et al. (2017) en simulaciones de Aspidosperma quebrachoblanco utilizaron valores de 16,8 pies ha $^{-1}$ año ${ }^{-1}$.

Crecimiento del volumen. Para la estimación de volumen y su distribución por clase, se recurrió a la función desarrollada por Chauchard (2009) en P. caldenia durante el último Inventario Forestal Nacional:

Va $=2,89421 *$ ga $-6,306998 *$ ga $^{1,5}+0,588971 *$ ga $*$ h $[18]$

Donde, Va: volumen con corteza hasta punta fina de $5 \mathrm{~cm}$ $\left(\mathrm{m}^{3}\right)$. ga: área basal acumulada de todos los fustes del árbol $\left(\mathrm{m}^{2}\right)$. h: altura total $(\mathrm{m})$.

La determinación de altura se realizó mediante el ajuste de la ecuación [19]:

$$
\mathrm{h}=12,59 *\left(1-0,8^{(-2,05 * \text { dap })}\right)
$$

Donde, h: altura total del árbol (m). dap: diámetro altura de pecho $(\mathrm{m})$.

Determinación de la cobertura arbórea. Para la determinación de la cobertura del estrato superior se ajustó la ecuación [20]:

$$
\mathrm{dc}=\frac{11,92}{\left(1+5,36^{(-6,44 * \mathrm{dap})}\right)}
$$

Donde, dc: diámetro de copa del árbol (m). dap: diámetro altura de pecho (m).
La ecuación [20] se aplicó a todos los pies de las parcelas para conseguir los diámetros de copas que luego se emplearon en la determinación de sus respectivas áreas, a fin de obtener el porcentaje de cobertura de la leñosa.

La ecuación [19] y [20] presentan un coeficiente de determinación $\mathrm{R}^{2}: 0,86$ y 0,65 , respectivamente. En ambas ecuaciones todos sus parámetros fueron altamente significativos $(P<0,0001)$ y sus respectivos coeficientes estimados se mostraron altamente correlacionados.

Análisis estadísticos. Los datos de biomasa herbácea fueron analizados mediante análisis de la varianza (ANOVA) y test LSD de Fisher para la comparación de medias, utilizando el software estadístico Infostat (Di Rienzo 2018). Se utilizó el mismo software para los análisis de regresión donde emplea el método de mínimos cuadrados en modelos lineales y el método Nelder-Mead simplex algorithm para las no lineales (Nelder y Mead 1965). La selección de los modelos se realizó comparando el coeficiente de determinación $\left(\mathrm{R}^{2}\right)$, el cuadrado medio del error (CMerror), el nivel de significancia de los parámetros, y los valores del criterio de información de Akaike (AIK) y el criterio de información bayesiano (BIC).

\section{RESULTADOS}

En cuanto a la disponibilidad de la biomasa herbáceas, de las cuatro situaciones evaluadas (cuadro 2), solo en la posición bajo copa en bosque cerrado se observó una disponibilidad significativamente inferior $(P<0,05)$. Es importante resaltar que en bosque abierto la cobertura de copas no superó el $50 \%$ de la superficie de suelo, esto puede apreciarse en la línea de corte en la figura 2.

Cuadro 2. Biomasa herbácea en kilogramos de materia seca (MS) área total y resumen de los principales indicadores de calidad de las pasturas en las cuatro situaciones estudiadas.

Herbaceous biomass in $\mathrm{kg}$ of dry matter (DM) total area and summary of the main indicators of pasture quality in the four situations

\begin{tabular}{|c|c|c|c|c|c|c|c|}
\hline \multicolumn{2}{|c|}{ Muestra } & Biomasa de & \multicolumn{5}{|c|}{ Indicadores de calidad del Forraje } \\
\hline $\begin{array}{l}\text { Tipo de } \\
\text { bosque }\end{array}$ & $\begin{array}{c}\text { Posición respecto } \\
\text { de la copa }\end{array}$ & MS kg ha-1 & $\begin{array}{c}\text { Proteína cruda } \\
\qquad(\%)\end{array}$ & $\begin{array}{c}\text { Digest. FDN } \\
(\%)\end{array}$ & $\begin{array}{l}\text { TND } \\
(\% \mathrm{MS})\end{array}$ & $\begin{array}{l}\mathrm{CNF} \\
(\% \mathrm{MS})\end{array}$ & VRF \\
\hline Abierto & Bajo copas & $2.923 \mathrm{a}$ & 13,3 & 38,2 & 52,7 & 18,3 & 88 \\
\hline Abierto & Entre copas & $2.732 \mathrm{a}$ & 11,3 & 32,2 & 52,0 & 17,7 & 79 \\
\hline Cerrado & Entre copas & $2.436 \mathrm{a}$ & 13,5 & 44,2 & 53,5 & 10,1 & 83 \\
\hline Cerrado & Bajo copas & $1.666 \mathrm{~b}$ & 14,5 & 48,5 & 55,3 & 17,1 & 91 \\
\hline \multicolumn{2}{|c|}{ Bosque $(P)$} & $0,0019^{*}$ & - & - & - & - & - \\
\hline \multicolumn{2}{|c|}{ Posición $(P)$} & $0,2286 \mathrm{~ns}$ & - & - & - & - & - \\
\hline \multicolumn{2}{|c|}{ Bosque x Posición $(P)$} & $0,0484^{*}$ & - & - & - & - & - \\
\hline
\end{tabular}
studied.

Análisis NIRS CV. Laboratorio certificado por National Forage Testing Association. VRF: valor relativo de forraje. CFN: carbohidratos no fibrosos. TND: total de nutrientes digestibles.

$P$ : probabilidades límites en ANOVA. *: $P<0,05$; ns: $P>0,05$. Letras diferentes indican diferencias significativas (LSD Fisher, $P<0,05$ ). 
En relación a la composición del pastizal, bajo copa en bosque cerrado, las muestras se integraban por un $40 \%$ de Setaria leucopila (Scribn. et Merr.) K.Schum, un $50 \%$ de Bromus catharticus Vahl y menos del $10 \%$ de Stipa hyalina Nees. En bosque cerrado, fuera de la proyección de copas, las muestras estuvieron integradas por un $50 \%$ de $S$. leucopila y un $50 \%$ de $S$. hyalina; no se encontró B. catharticus. En el bosque abierto, bajo la proyección de copas, las muestras estuvieron integradas por un $10 \%$ de S. leucopila, un $10 \%$ de B. catharticus, un $10 \%$ de Nassella tenuissima Barkworth y un $70 \%$ de Carex sororia Kunth. En la posi- ción de bosque abierto entre copas, las muestras presentaron un $10 \%$ de Stipa brachychaeta Godron, un $20 \%$ de $S$. leucopila y un $80 \%$ de Cyperus cayennensis Britton.

Los resultados del componente leñoso muestran que al comparar la distribución de $\mathrm{AB}$ actual versus ideal, por clase diamétrica, se observa ciertos baches que podrían comprometer la producción sostenida en el tiempo (figura 1). La distribución ideal planteada buscó la recuperación de la estructura irregular con una mayor participación de pies jóvenes en la distribución de pies por clase diamétrica (cuadro 3).

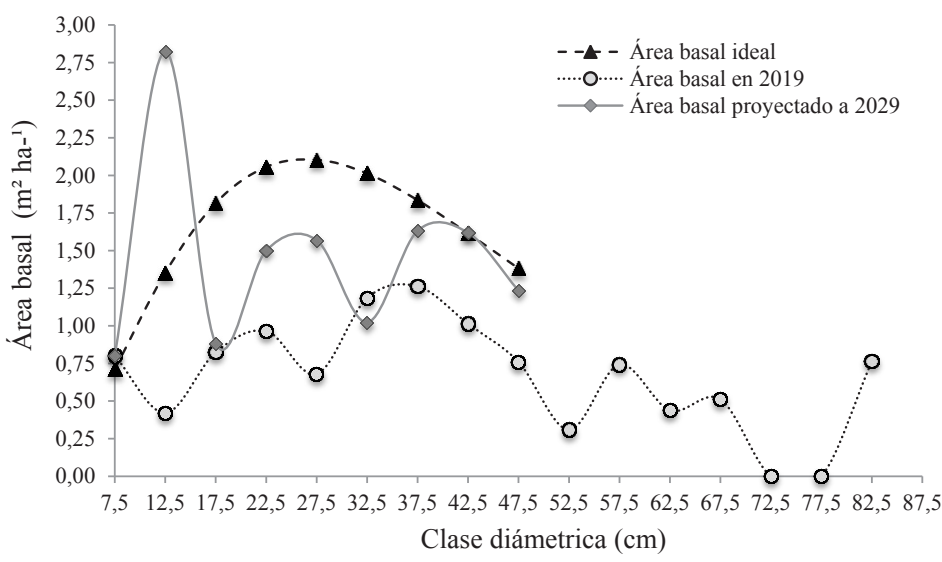

Figura 1. Distribución del área basal $\left(\mathrm{m}^{2} \mathrm{ha}^{-1}\right)$ : actual, ideal y proyectada en función de la clase diamétrica (cm).

Distribution of the basal area $\left(\mathrm{m}^{2} \mathrm{ha}^{-1}\right)$ : current, ideal and projected according to the diametric class $(\mathrm{cm})$.

Cuadro 3. Proyección del número de pies, área basal y volumen total por clase diamétrica para un periodo de 10 años.

Projection of the number of feet, basal area and total volume by diameter class for a period of 10 years.

\begin{tabular}{|c|c|c|c|c|c|c|c|c|c|c|}
\hline \multirow[b]{2}{*}{$\begin{array}{l}\text { Clase } \\
(\mathrm{cm})\end{array}$} & \multirow[b]{2}{*}{$\begin{array}{c}\text { *Incremento } \\
\text { Corriente }\left(\mathrm{mm} \text { año } \mathrm{o}^{-1}\right)\end{array}$} & \multicolumn{3}{|c|}{ Frecuencia $\left(\mathrm{N}\right.$ pies ha $\left.{ }^{-1}\right)$} & \multicolumn{3}{|c|}{ Área basimétrica $\left(\mathrm{m}^{2} \mathrm{ha}^{-1}\right)$} & \multicolumn{3}{|c|}{ Volumen $\left(\mathrm{m}^{3} \mathrm{ha}^{-1}\right)$} \\
\hline & & $\begin{array}{l}\text { Año } \\
2029\end{array}$ & Ideal & Diferencia & 2029 & $\begin{array}{l}\text { Año } \\
\text { Ideal }\end{array}$ & Diferencia & $\begin{array}{l}\text { Año } \\
2029\end{array}$ & Ideal & Diferencia \\
\hline 2,5 & 5,1 & 234,0 & 235,0 & $-1,0$ & 0,11 & 0,12 & 0,00 & 0,52 & 0,52 & 0,00 \\
\hline 7,5 & 6,6 & 181,5 & 161,0 & 20,6 & 0,80 & 0,71 & 0,09 & 3,85 & 3,42 & 0,44 \\
\hline 12,5 & 6,2 & 230,0 & 110,2 & 119,7 & 2,82 & 1,35 & 1,47 & 14,17 & 6,79 & 7,38 \\
\hline 17,5 & 5,9 & 36,7 & 75,5 & $-38,8$ & 0,88 & 1,82 & $-0,93$ & 4,58 & 9,42 & $-4,84$ \\
\hline 22,5 & 5,5 & 37,7 & 51,7 & $-14,0$ & 1,50 & 2,06 & $-0,56$ & 7,97 & 10,92 & $-2,96$ \\
\hline 27,5 & 5,2 & 26,4 & 35,4 & $-9,0$ & 1,57 & 2,10 & $-0,53$ & 8,47 & 11,35 & $-2,89$ \\
\hline 32,5 & 4,8 & 12,3 & 24,3 & $-11,9$ & 1,02 & 2,01 & $-0,99$ & 5,57 & 10,96 & $-5,39$ \\
\hline 37,5 & 4,6 & 14,8 & 16,6 & $-1,8$ & 1,63 & 1,84 & $-0,20$ & 8,91 & 10,03 & $-1,11$ \\
\hline 42,5 & 4,3 & 11,4 & 11,4 & 0,0 & 1,62 & 1,61 & 0,00 & 8,82 & 8,80 & 0,02 \\
\hline 47,5 & 4,1 & 7,0 & 7,8 & $-0,8$ & 1,23 & 1,38 & $-0,15$ & 6,68 & 7,48 & $-0,80$ \\
\hline & Total ha- ${ }^{-1}$ & 792 & 729 & 63 & 13,2 & 15,0 & $-1,8$ & 69,5 & 79,7 & $-10,2$ \\
\hline
\end{tabular}

Los cálculos de la distribución ideal de pies, $\mathrm{AB}$ y volumen, son en base a un valor de q: 1,46, un $\mathrm{AB}$ total de $15 \mathrm{~m}^{2} \mathrm{ha}^{-1}$ y un diámetro máximo de corta de $50 \mathrm{~cm}$. El número de pies, AB y volumen proyectados para el año 2029 resulta de la aplicación del IC calculado por clase y la tasa de reclutamiento. Los valores negativos en la columna "diferencia", indican un déficit y los valores positivos el exceso con respecto a la curva balanceada que se ha determinado. 
Por otro lado, la proyección del crecimiento a partir de la distribución diamétrica del bosque abierto, permite observar un aumento considerable de densidad en la clase diamétrica de 12,5 cm. Este aumento representaría el $108 \%$ en el número de pies y área basal (cuadro 3) con respecto a la distribución ideal o de equilibrio establecida como distribución óptima de manejo. Es importante remarcar que se habla de un aumento fuera del óptimo, porque según lo establecido, con 110 pies en la clase $12,5 \mathrm{~cm}$ para el año 2029 , sería suficiente para asegurar la reserva de la clase siguiente de $17,5 \mathrm{~cm}$.

La relación entre la cobertura copas y el área basal mostró una alta asociación (figura 2) expresado en un coeficiente de determinación $\mathrm{R}^{2}=0,96$. El aumento en la cobertura de copas fue de $3,18 \%$ por unidad de incremento de $\mathrm{AB}\left(\mathrm{m}^{2} \mathrm{ha}^{-1}\right)(\mathrm{EE}=0,10$; $\min .2,96(95 \%)$ y máx. $3,39(95 \%) ; \mathrm{n}=39)$. Este resultado permite transformar las magnitudes de densidad en área basal a porcentaje de cobertura. Esto toma gran relevancia ya que, el tratamiento de la densidad en la bibliografía aparece expresada en área basal, desde un abordaje netamente forestal, y en términos cualitativos desde un punto de vista ganadero o del manejo del pastizal.

Si asociamos los resultados de la simulación del vuelo forestal con la relación de cobertura de copas y área basal (figura 2), el incremento en densidad de $1,47 \mathrm{~m}^{2} \mathrm{ha}^{-1}$ en la clase de $12,5 \mathrm{~cm}$, se traduciría en un $5 \%$ de cobertura arbórea excedente al óptimo para mantener una estructura exponencial negativa o en forma de J invertida. Además, la

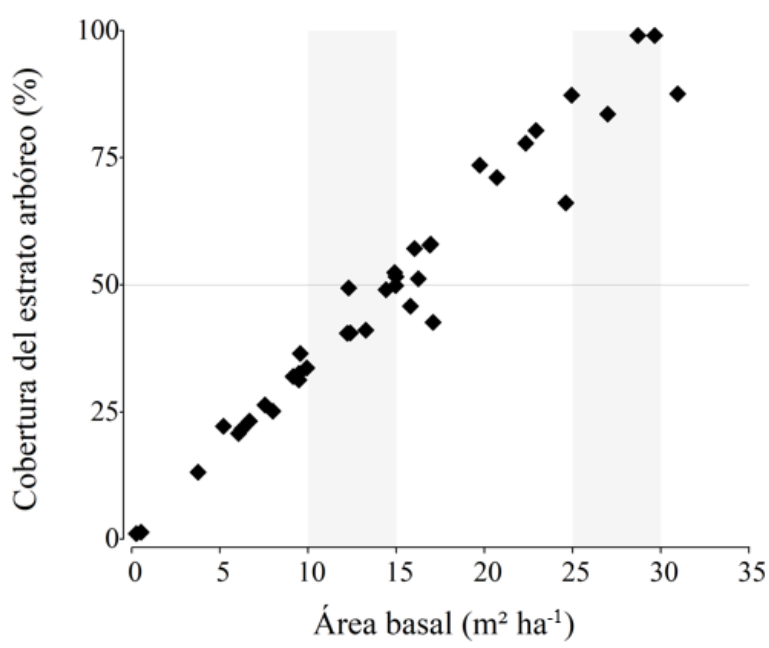

Figura 2. Relación entre la cobertura del estrato arbóreo y el área basal para cada parcela de muestreo. Bandas grises corresponden a la densidades en términos de área basal arbórea donde se realizaron los muestreos de biomasa herbácea.

Relationship between the coverage of the tree stratum and the basal area for each sample plot. Gray bands correspond to the densities in terms of the tree basal area where herbaceous biomass samplings were carried out. relación mencionada (figura 2), permite estimar que, con un aprovechamiento por diámetro mínimo de corta, si se remueve la última clase diamétrica ( 7 pies), que equivale a $1,23 \mathrm{~m}^{2} \mathrm{ha}^{-1}$, se liberarían un $4 \%$ de la superficie del suelo.

\section{DISCUSIÓN}

Los resultados del componente herbáceo hacen suponer que, para mantener los niveles de producción de biomasa forrajera al máximo, debería manejarse el estrato arbóreo en niveles de 10 a $15 \mathrm{~m}^{2}$ ha-1 de $\mathrm{AB}$, que fue el rango en el que se inventarió la biomasa herbácea en bosque abierto (figura 2) ya que, en la condición bajo las copas en el bosque cerrado la disponibilidad de biomasa herbácea es significativamente menor $(P<0,05)$, respecto al resto de las posiciones. Esto indicaría que, en el bosque abierto los niveles de sombra o competencia por agua no son tan marcados (relación entre el $\mathrm{AB}$ acumulada y la cobertura de copas, figura 2) y, por lo tanto, la disponibilidad de biomasa herbácea no difiere bajo o fuera de la proyección de copas.

Un aspecto a tener en cuenta es que, la inclinación de los rayos solares genera que las sombras proyectadas por la mañana temprana, y a medida que avanza la tarde, sean más largas que las proyectadas al mediodía. Debido a esto, en esos momentos del día, bajo la copa de los árboles no se proyecta la sombra producida por el propio árbol, aunque si pueden proyectarse sombras de algún otro árbol adyacente. Esta última situación es más probable a medida que aumenta el AB (Fiandino 2019). Por otro lado, si bien la radiación fotosintéticamente activa bajo el dosel de la leñosa no se midió, muchos autores y para diferentes especies, encuentran que el límite para la producción de forraje ronda entre el $70 \%$ (Fernández et al. 2002) y el $50 \%$ (Fiandino 2019) de la interceptación de la radiación. Esto permite deducir que, bajo copas en bosque abierto, la disminución de la radiación no supera dicho umbral (observar línea de corte en $50 \%$ de cobertura en la figura 2).

En cuanto a la calidad del forraje, si bien no se analizó estadísticamente, los valores alcanzados en las situaciones evaluadas (cuadro 2) puede atribuirse a las diferentes comunidades vegetales que allí crecen. La posición bajo copa en bosque cerrado, las muestras estaban integradas principalmente por especies citadas como de mayor calidad forrajera. Gaggiotti et al. (1996) determinaron que $B$. catharticus alcanza una digestibilidad máxima de $77 \%$. Por ende, la presencia de B. catharticus bajo el dosel de los árboles, tendería a mejorar la calidad de la base forrajera.

La mayor proporción de $S$. leucopila y B. catharticus en bosque cerrado, coincide con lo encontrado por Gabutti et al. (1999), donde reporta un aumento de estas especies en el sotobosque en pastizales bajo caldenales en la provincia de San Luis, Argentina. En el mismo sentido, el aumento de estas especies bajo la proyección de copas de $P$. caldenia, tienden a mejorar la calidad del pastizal.

En bosque abierto, bajo la proyección de copas, también aparece $B$. catharticus aunque en muy baja propor- 
ción. Se observa un $70 \%$ de C. sororia, de una digestibilidad máxima de $58 \%$ (Hidalgo et al. 1998) que hace que la calidad de la muestra sea superior a la situación de bosque abierto donde comienza a aparecer especies como S. brachychaeta, de escaso valor forrajero.

En cuanto al componente leñoso, la distribución diamétrica desequilibrada que caracteriza al bosque en la actualidad es, probablemente, el resultado de regímenes de aprovechamiento que fueron implementados en el pasado sin un criterio silvícola claro. La proyección de su crecimiento en los próximos 10 años, representan un amento en la densidad en términos de $\mathrm{AB}$ que, a su vez, se traduce en un amento en la cobertura de copas. Esto significa que, de no considerar el manejo de estos excedentes, llevaría a un cierre del dosel, una disminución de los incrementos diamétricos y a una menor producción de pasto cuando se exceda el $50 \%$ de cobertura arbórea.

El modelado de crecimiento forestal permite anticiparse y visualizar como se incrementará el número de pies en las sucesivas clases diaméntricas, lo que posibilitaría planificar cortas de mejoramiento como: clareos, raleos, cortas de liberación, limpieza o sanitarias, que permitan hacer una selección de los individuos de futura cosecha y, proyectar el número de pies y volumen de madera a obtener en un determinado periodo.

Los resultados de volumen total proyectados a 2029 $\left(69,5 \mathrm{~m}^{3} \mathrm{ha}^{-1}\right)$ concuerda con los datos proporcionados por el último inventario forestal nacional (SAyDS 2007), donde en bosques de $P$. caldenia de $15 \mathrm{~m}^{2}$ ha ${ }^{-1}$ de AB, se reportó un volumen medio total de $62,3 \mathrm{~m}^{3} \mathrm{ha}^{-1}$. Estos valores de producción se encuentran en concomitancia con los reportados por Coirini et al. (2013), donde en el distrito del $P$. caldenia es posible obtener entre 0,5 y $0,8 \mathrm{~m}^{3} \mathrm{ha}^{-1}$ de incremento anual. Si multiplicamos estos valores por el periodo proyectado tenemos entre 5 y $8 \mathrm{~m}^{3} \mathrm{ha}^{-1}$, lo que significa que los resultados de la simulación están dentro de los encontrado por otros autores en bosques de $P$. caldenia. En síntesis, para la estructura bajo estudio, si el silvicultor opta por cortar la última clase, en el año 2029, obtendrían siete pies maduros que se encuentran en la clase de 47,5 $\mathrm{cm}$, lo que equivale a $6,68 \mathrm{~m}^{3} \mathrm{ha}^{-1}$. El volumen acumulado por todas las clases diamétricas será de $69,5 \mathrm{~m}^{3} \mathrm{ha}^{-1}$, lo que significaría remover tan solo el 9,61 \% del volumen total en pie. En masas irregulares es recomendable no remover más del $20 \%$ (Araujo 2014) para no tender a su regularidad y mantener la estructura de equilibrio.

Por último, en términos de cobertura de copas, la remoción de la última clase liberaría un $4 \%$ de la superficie, mientras que aplicar un raleo con una intensidad del $50 \%$ de los pies en la clase de $12,5 \mathrm{~cm}$, establecido de acuerdo a la distribución ideal, permitiría liberar un $5 \%$ de la superficie. Por lo tanto, la experiencia de este análisis nos pone de manifiesto en términos relativos los impactos que tendrían las prácticas silvícolas de mejoramiento y aprovechamiento sobre la apertura del vuelo forestal. La cual, a su vez, impactará en el desempeño de la herbácea.

\section{CONCLUSIONES}

En relación al componente herbáceo se concluye que, hasta ciertos niveles de área basal acumulada, no se producen disminuciones significativas en la disponibilidad de biomasa forrajera bajo la proyección de copas, respecto la posición a cielo abierto, lo que permitiría manejar el dosel de la leñosa en los niveles indicados. Además, bajo la proyección de las copas, se observó un cambio en la composición del pastizal a favor de especies de mayor valor forrajero y que sería interesante de indagar en profundidad en futuras investigaciones ya que, hasta aquí, no se puedo concluir con evidencia suficiente.

En cuanto al componente forestal, la experiencia de este trabajo indica que el empleo de un modelo de clases diamétricas arroja resultados satisfactorios en cuanto a la proyección de los parámetros forestales, contrastados a valores reportados en bosque de similares características, lo que permitirían planificar las cortas de mejoramientos y conducir estos bosques en niveles de cobertura que no afecten significativamente el rendimiento del recurso forrajero.

La estrecha relación entre la cobertura de copas y el área basal permite transformar los valores normalmente expresados en área basal a porcentaje de cobertura. Además, dicha relación apoyada mediante un método de simulación, como el aquí desarrollado, pueden predecir cómo evolucionaría la cobertura arbórea en el tiempo y el impacto de las prácticas silvícolas para mantener una estructura diamétrica objetivo y que, a su vez, maximice el rendimiento del componente herbáceo.

Las limitantes de este trabajo están relacionadas a la modelación en dos aspectos. Uno tiene que ver con la tasa de mortalidad y, el otro, con la tasa de reclutamiento. $\mathrm{Si}$ bien, en un principio, se estimó mediante la construcción de índices por información recabada de los inventarios, luego se decidió no implementarlos y se optó por no extender el periodo de simulación más allá de 10 años, como lo sugieren los autores de estos modelos. La tarea que resta hacia adelante es, a través de parcelas permanentes, medir tasas de mortalidad, reclutamientos e incrementos diamétricos asociados a variables de sitio para validar y mejorar la capacidad de predicción de los modelos.

\section{AGRADECIMIENTOS}

Deseo expresar mi profundo agradecimiento al Med. Vet. Daniel Horacio Freires encargado del establecimiento y al Ing. Agr. Emiliano Freires encargado del módulo experimental silvopostaril. Ambos, en todo momento, pusieron a disposición las instalaciones y los recursos económicos necesarios para los análisis de calidad del forraje. Además, hay que resaltar que, son los impulsores y encargados de llevar adelante una propuesta de aprovechamiento sostenible del bosque, sin la utilización de forrajeras exóticas y sin la intervención mediante prácticas destructivas de la 
regeneración del estrato arbóreo, lo que posibilitaría avanzar con la metodología de ordenación de bosque irregular propuesta en este trabajo.

\section{REFERENCIAS}

Anderson DL, JA Del Águila, AE Bernardón. 1970. Las formaciones vegetales en la provincia de San Luis. Revista de Investigaciones Agropecuarias. Serie 2. Biología y Producción Vegetal 2(3): 153-183.

Araujo PA, MC Iturre. 2014. Ordenamiento de Masas Irregulares. Facultad de Ciencias Forestales. Universidad Nacional de Santiago del Estero. E-Book ISBN 978-987-1676-39-2. Consultado 10 jul. 2018. Disponible en: https://fcf.unse. edu.ar/archivos/series-didacticas/SD-28-Ordenacion-bosques-irregulares-ARAUJO.pdf

Beltrán HA, L Chauchard, A Dezzotti, GM Pastur. 2018. Modelo de crecimiento diamétrico de Nothofagus alpina y su relación con el de Nothofagus obliqua y Nothofagus dombeyi en los bosques naturales de la Patagonia argentina. Bosque 39(1): 107-117. http://dx.doi.org/10.4067/S071792002018000100107.

Bogino S, R Villalba. 2008. Radial growth and biological rotation age of Prosopis caldenia Burkart in Central Argentina. Journal of Arid Environments 72: 16-23. https://doi. org/10.1016/j.jaridenv.2007.04.008.

Chauchard L, R Sbrancia, A Medina, A Rabino. 2009. Funciones de Volumen Total para Prosopis caldenia Burk, Argentina. Quebracho 17(1,2): 41-51.

Coirini R, M Karlin, M Brassiolo. 2013. Prácticas forestales en los bosques nativos de la República Argentina Ecorregión Forestal Espinal. Buenos Aires, Argentina. Secretaría de Ambiente y Desarrollo Sustentable de la Nación Argentina. $175 \mathrm{p}$.

Di Rienzo JA, F Casanoves, MG Balzarini, L Gonzalez, M Tablada, CW Robledo. 2018. Grupo InfoStat, FCA, Universidad Nacional de Córdoba, Argentina. http://www.infostat.com.ar

Dussart E, Lerner P, Peinetti R. 1998. Long term dynamics or two populations on Prosopis caldenia Burkart. Journal of Range Management 51: 685-691.

Estelrich HD, CC Chirino, EF Morici, B Fernández. 2005. Dinámica de áreas naturales cubiertas por bosque y pastizal en la Región semiárida central de la Argentina. Modelo conceptual. Heterogeneidad de la Vegetación. Santa Rosa, La Pampa, Argentina. Facultad de Agronomía UNLPam. p. 351-364.

Fernández ME, JE Gyenge, G Dalla-Salda, T Schlichter. 2002. Silvopastoral systems in NW Patagonia: I. Growth and photosynthesis of Stipa speciosa under different levels of Pinus ponderosa cover. Agroforestry Systems 55: 27-35.

Fiandino SI, 2019. Efecto de los recursos agua y radiación sobre la productividad del pastizal natural en sistemas silvopastoriles de la Sierra de Comechingones. Tesis Doctoral. Universidad Nacional de Río Cuarto. 246 p.

Gabutti EG, Privitello MJL, Maidana MA, Harrison RU. 1999. Producción anual del pastizal natural del bosque de cal- dén (Prosopis caldenia burk.) de la provincia de San Luis, Argentina. Archivo Latinoamericano Producción Animal 7(1): $1-8$.

Gaggiotti MdelC, LA Romero, OA Bruno, EA Comeron, OR Quaino. 1996. Tabla de composición química de alimentos. II - Forrajes verdes. INTA. Rafaela, Santa Fe, Argentina.

Hidalgo LG, MA Cauhépé, AN Erni. 1998. Digestibilidad y contenido de proteína bruta en especies de pastizal de la Pampa Deprimida. Argentina. Revista de Investigación Agraria: Producción y Sanidad Animales 13: 165-177.

Iturre MC, PA Araujo, CJ Trejo. 2017. Simulación del crecimiento de bosques nativos del Chaco Semiarido. Aplicación del sistema informático MOSIMAFO. Quebracho 25(1,2): 54-62.

Madrigal CA. 1994. Ordenación de montes arbolados. Madrid, Esàña. ICONA. 375 p.

Morici E, V Doménech-García, G Gómez-Castro, A Kin, A Saenz, C Rabotnikof. 2009. Diferencias estructurales entre parches de pastizal del caldenal y su influencia sobre el banco de semillas, en la provincia de la pampa, argentina. Agrociencia 43: 529-537.

Nelder JA, R Mead. 1965. A simplex method for function minimization. The computer journal 7(4): 308-313.

Pelaez D, RM Boo, OR Elia. 1992. Emergence and seedling survival of caldén in the semiarid region of Argentina. Journal of Range Management 45: 564-568. http://hdl.handle. net $/ 10150 / 644555$

Roman L, C Fuser, M Cocco, M Flores Palenzona, C Percara, C de la Peña. 2015. Evaluación del componente herbáceo en el espinal del noroeste del departamento Concordia, Entre Ríos. In $3^{\circ}$ Congreso Nacional de Sistemas Silvopastoriles: VIII Congreso Internacional Sistemas Agroforestales. Iguazú, Argentina. Ediciones INTA. p. 37-38.

Sanquetta CR, JE Arce, F dos Santos Gomes, E Coutinho da Cruz. 1999. Evaluación y simulación precoces del crecimiento de rodales de Pinus taeda L. con matrices de transición. Quebracho 7: 31-42.

SAyDS (Secretaría de Ambiente y Desarrollo Sustentable de la Nación Argentina, AR). 2007. Primer inventario nacional de bosques nativos: informe regional espinal, segunda parte. Buenos Aires, Argentina. Secretaría de Ambiente y Desarrollo Sustentable de la Nación Argentina. 154 p.

Silva JNM. 1989. The behaviour of the tropical rain forest of the brasilian amazon after logging. Tesis Ph.D. Oxford, Inglaterra. Oxford Forestry Institute, University of Oxford. $119 \mathrm{p}$.

Urbano E, S do Amaral Machado, AF Filho, CR Sanquetta, JD Zea-Camaño. 2018. Modelación del volumen de rodal para especies secundarias en bosques nativos de Mimosa scabrella en la región metropolitana de Curitiba, Paraná, Brasil. Bosque 39(2): 227-237. http://dx.doi.org/10.4067/S0717$\underline{92002018000200227}$

Vargas BL, JR Corral, FC Cruz, OC Aguirre, J Nagel. 2008. Uso y aplicación de los simuladores de crecimiento forestal en la toma de decisiones silviculturales. Revista Forestal Latinoamericana 23(2): 33-52. http://www.saber.ula.ve/handle/123456789/33374 